\title{
The pancake-bonding of semiquinone radicals under variable pressure and temperature conditions
}

\author{
Nikita E. Bogdanov ${ }^{1,2^{*}}$, Valentina Milašinović ${ }^{3}$, Boris Zakharov ${ }^{1,2}$, Elena V. Boldyreva ${ }^{1,2}$, Krešimir Molčanov $^{3}$ \\ ${ }^{1}$ Boreskov Institute of Catalysis, 5 Ac. Lavrentieva avenue, 630090 Novosibirsk, Russia, ${ }^{2}$ Novosibirsk State University, 2 Pirogova str. \\ 2, 630090 Novosibirsk Russia, ${ }^{3}$ Ruđer Bošković Institute, Bijenička 54, 10000 Zagreb, Croatia. \\ *bogdanovne@catalysis.ru
}

In this work the effects of pressure and temperature on the nonlocalized two-electron multicentric covalent bonds ('pancake bonding') in closely bound radical dimers were probed by single-crystal X-ray diffraction on a 4-cyano-N-methylpyridinium salt of 5,6-dichloro-2,3dicyanosemiquinone (DDQ.4CN) and I-methylpyridinium salt of tetrabromosemiquinone radical anion $\left(\mathrm{Br}_{4} \mathrm{Q} \cdot \mathrm{NMePyr}\right)$ as the sample compounds.

The DDQ.4CN crystal structure can be described as closely bound stacked dimers of radical anions with interplanar separation $<3.2 \AA$, which is known as non-localized two electron covalent bonding. At ambient conditions the stacks of pancake bonded radical anions are formed by two types of distances: short intra-dimer and long inter-dimer contacts. On cooling, the anisotropic structural compression was accompanied by continuous changes in molecular stacking; the discontinuities in the changes in volume and $b$ and $c$ cell parameters suggest that a phase transformation occurs between 210 and $240 \mathrm{~K}$. At a pressure of $2.55 \mathrm{GPa}$, both distances between radical dimers shortened to $2.9 \AA$, and become roughly equal, which corresponds to distances observed in extended-bonded polymers. Increasing pressure further to $6 \mathrm{GPa}$ reduced the interplanar separation of the radicals to $2.75 \AA$, which may indicate that the covalent component of the interaction significantly increased [1]. The linear strain analysis shows that the most deformations of pressure and temperature occurs in the direction of pancake bonding.

The $\mathrm{Br}_{4} \mathrm{Q} \cdot N \mathrm{MePyr}$ crystal structure is built of infinite stacks of equidistant radical anions with no Peierls distortion [2]. On cooling the structure is compressed monotonically, the distance between radicals changes non-linearly, compress to $<3.3 \AA$, but the space group remains the same. Upon pressure, the structure is compressed monotonically with no phase transformations in all the pressure range $(0$ - 6.0 GPa), the lowest interplanar distance is $<2.9 \mathrm{GPa}$, that may indicate the increasing of the covalent component in pancake bond and a significant decrease of the electron jumping barrier which may influence semiconductivity.

[1] Bogdanov, N. E., Milašinović, V., Zakharov, B. A., Boldyreva, E. V. \& Molčanov, K. (2020). Acta Crystallogr. Sect. B Struct. Sci. Cryst. Eng. Mater. 76, 285-291.

[2] Molčanov, K., Stilinović, V., Šantić, A., Maltar-Strmečki, N., Pajić, D. \& Kojić-Prodić, B. (2016). Cryst. Growth Des. 16, $4777-4782$.

Keywords: multicentric two-electron bonding, high-pressure, low temperature, X-ray diffraction

The equipment of Research and Education Center "Molecular design and ecologically safe technologies" (NSU). NEB, BAZ, EVB acknowledge support by the Ministry of Science and Higher Education of Russia; the project was carried out jointly by Boreskov Institute of Catalysis (governmental order AAAA-A21-121011390011-4) and Novosibirsk State University. 